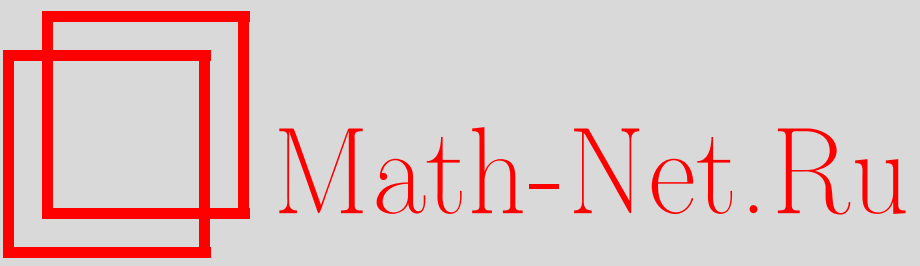

А. Е. Миловидов, Г. С. Шаров, Замкнутые релятивистские струны в пространствах с нетривиальной геометрией, ТМФ, 2005, том 142, номер 1, 72-82

DOI: https://doi.org/10.4213/tmf1765

Использование Общероссийского математического портала Math-Net.Ru подразумевает, что вы прочитали и согласны с пользовательским соглашением

http://www . mathnet.ru/rus/agreement

Параметры загрузки:

IP : 34.229 .45 .116

26 апреля 2023 г., 15:15:28 
ТЕОРЕТИЧЕСКАЯ

И МАТЕМАТИЧЕСКАЯ

ФИЗИКА

Том 142, № 1

январь, 2005

(C) 2005 г.

А.Е. Миловидов*, Г. С. Шаров*

\section{ЗАМКНУТЫЕ РЕЛЯТИВИСТСКИЕ СТРУНЫ В ПРОСТРАНСТВАХ С НЕТРИВИАЛЬНОЙ ГЕОМЕТРИЕЙ}

Найдены точные решения уравнений движения для замкнутой релятивистской струны, которая нагружена точечной массой и движется в пространстве, являющемся прямым произведением пространства Минковского на компактное многообразие (многомерный тор). Исследованы физические характеристики состояний системы, описываемых этими решениями.

Ключевые слова: замкнутая релятивистская струна с точечной массой, компактное многообразие.

Свободная от квантовых аномалий теория струн [1], [2] существует в пространстве-времени критической размерности $D$. В частности, $D=26$ для бозонной струны и $D=10$ для струны с фермионными степенями свободы. Попытки решения проблемы лишних измерений имеют целью создание теории без аномалий при $D=4$ [3] или разработку процедуры компактификации исходного $D$-мерного пространства Минковского $R^{1, D-1}$ в многообразие $\mathcal{M}=R^{1,3} \otimes K$. Последнее включает компактное многообразие $K$ размерности $D-4$, характерный размер которого достаточно мал [1].

В настоящей работе мы рассматриваем динамику замкнутой струны в многообразии $\mathcal{M}=R^{1,3} \otimes K$ (являюшемся результатом компактификации), не уточняя саму процедуру компактификации. В качестве компакта $K$ выступает $(D-4)$-мерный тор: $K=S^{1} \otimes$ $S^{1} \otimes \cdots \otimes S^{1}[1]$

Рассмотрим в пространстве $\mathcal{M}$ замкнутую релятивистскую струну с одной точечной массой $m$. Эта система описьвается действием

$$
S=-\gamma \int_{\Omega} \sqrt{-g} d \tau d \sigma-m \int \sqrt{\dot{x}_{1}^{2}(\tau)} d \tau
$$

Здесь $\gamma$ - натяжение струны, $g$ - определитель индуцированной метрики

$$
g_{a b}=G_{\mu \nu}(X) \partial_{a} X^{\mu} \partial_{b} X^{\nu}
$$

* Тверской государственный университет, Тверь, Россия.

E-mail: s002027@tversu.ru, german.sharov@tversu.ru 
на мировой поверхности струны $X^{\mu}(\tau, \sigma)$, погруженной в $\mathcal{M}, G_{\mu \nu}(X)$ - метрика на $\mathcal{M}$, $\Omega=\left\{\tau, \sigma: \tau_{1}<\tau<\tau_{2}, \sigma_{1}(\tau)<\sigma<\sigma_{2}(\tau)\right\} ;$ уравнения $x_{i}^{\mu}(\tau)=X^{\mu}\left(\tau, \sigma_{i}(\tau)\right), i=1,2$, описывают одну и ту же траекторию массивной точки струны на мировой поверхности, имеюшей форму трубки [4]:

$$
x_{1}^{\mu}(\tau)=X^{\mu}\left(\tau, \sigma_{1}(\tau)\right) \stackrel{\cong}{\cong} X^{\mu}\left(\tau^{*}, \sigma_{2}\left(\tau^{*}\right)\right)=x_{2}^{\mu}\left(\tau^{*}\right) .
$$

В параметризации этой линии могут участвовать два различных параметра $\tau$ и $\tau^{*}$, связанных соотношением $\tau^{*}=\tau^{*}(\tau)$, которым следует дополнить условие замыкания $(2)$ мировой поверхности. Смысл символа равенства $\cong$ в условии (2) для координат, относящихся к компакту $K$, будет уточнен ниже.

Замкнутая струна с точечной массой, гомеоморфная окружности с выделенной точкой, может служить моделью точечной (элементарной) частишы, обладаюшей определенными внутренними степенями свободы. Эти степени свободы играют ту же роль, что и струнные возбуждения в струнных моделях адронов [2], [4]-[6], в модели "протяженная частица" [7] и в других струнных моделях. Особенностью рассматриваемой модели является нетривиальная фундаментальная группа пространства $\mathcal{M}$, содержашего компактное подмногообразие $K$. Вследствие этого замкнутая струна может относиться к тому или иному гомотопическому классу $\mathcal{M}$.

Полученные с помощью варьирования действия (1) уравнения движения системы без потери обшности можно свести к простейшему виду [4], если выбрать параметры $\tau$ и $\sigma$ так, чтобы были выполнены условия ортонормальности

$$
\left(\partial_{\tau} X \pm \partial_{\sigma} X\right)^{2}=0
$$

а также условия

$$
\sigma_{1}(\tau)=0, \quad \sigma_{2}(\tau)=2 \pi
$$

Скалярньй квадрат в условиях ортонормальности (3) понимается в смысле скалярного произведения $(\xi, \zeta)=G_{\mu \nu} \xi^{\mu} \zeta^{\nu}$.

Для замкнутой струны без масс наряду с условиями (3) мы можем без ограничения обшности потребовать выполнения равенства $\tau=\tau^{*}$ в условии замыкания (2). Однако для струны с действием (1) при $m \neq 0$ из-за наличия выделенной (массивной) точки не на всякой мировой поверхности достижимы равенства $\tau=\tau^{*},(3)$ и (4).

При выполнении условий (3) (означающих, что индуцированная метрика $g_{a b}$ является конформно-плоской) уравнения движения струны принимают вид

$$
\frac{\partial^{2} X^{\mu}}{\partial \tau^{2}}-\frac{\partial^{2} X^{\mu}}{\partial \sigma^{2}}+\Gamma_{\lambda \nu}^{\mu}(X)\left(\frac{\partial X^{\lambda}}{\partial \tau} \frac{\partial X^{\nu}}{\partial \tau}-\frac{\partial X^{\lambda}}{\partial \sigma} \frac{\partial X^{\nu}}{\partial \sigma}\right)=0
$$

где $\Gamma_{\lambda \nu}^{\mu}(X)$ - символы Кристоффеля в $\mathcal{M}$.

Рассматривая в качестве компакта $K$ тор размерности $D-4$, будем предполагать, что тор $K$ и многообразие $\mathcal{M}=R^{1,3} \otimes K$ являются плоскими, и введем координаты $x^{\mu}$ так, что $x^{0}, x^{1}, x^{2}$ и $x^{3}$ относятся к $R^{1,3}$, а координаты, описываюшие $K$ (ниже мы будем обозначать их $\left.x^{k}, k=4,5, \ldots\right)$, являются циклическими с периодом $\ell_{k}$. Последнее означает, что точки с координатами $x^{k}$ и $x^{k}+N_{k} \ell_{k}, N_{k} \in \mathbb{Z}$, отождествлены: $x^{k} \cong x^{k}+N \ell_{k}$. 
Равенство с учетом данной факторизации фигурирует в условии замыкания (2). Принимая во внимание равенства (4), перепишем условие (2) в виде

$$
X^{\mu}\left(\tau^{*}, 2 \pi\right)=X^{\mu}(\tau, 0)+\sum_{k} N_{k} \ell_{k} \delta_{k}^{\mu}
$$

где $\delta_{k}^{\mu}-$ символ Кронекера.

Метрика данного многообразия $\mathcal{M}$ совпадает с метрикой пространства Минковского $R^{1, D-1}: G_{\mu \nu}=\eta_{\mu \nu}=\operatorname{diag}(1,-1, \ldots,-1)$. Следовательно, в уравнении $(5) \Gamma_{\lambda \nu}^{\mu}=0$, и уравнение линеаризуется.

Однако краевое условие на траектории массивной точки (уравнение ее движения), выводимое из действия (1), остается нелинейным:

$$
m \frac{d}{d \tau} \frac{\dot{X}^{\mu}(\tau, 0)}{\sqrt{\dot{X}^{2}(\tau, 0)}}+\gamma\left[X^{\prime \mu}\left(\tau^{*}, 2 \pi\right)-X^{\prime \mu}(\tau, 0)\right]=0
$$

где $\dot{X}^{\mu} \equiv \partial_{\tau} X^{\mu}, X^{\prime \mu} \equiv \partial_{\sigma} X^{\mu}$.

Итак, динамика замкнутой релятивистской струны с точечной массой в пространстве $\mathcal{M}$ описывается системой уравнений (3)-(7). Будем искать решения этой системы, допускающие линеаризацию краевого условия (7) в рамках условий ортонормальности (3). Для этого используем подход, развитый в работе [8] для струны с массивными концами. Наложим ограничения

$$
\sqrt{\dot{X}^{2}(\tau, 0)}=C=\text { const }, \quad \tau^{*}=\tau+\tau_{0}, \quad \tau_{0}=\text { const }
$$

После этого уравнения движения сводятся к системе уравнений

$$
\begin{gathered}
\frac{\partial^{2} X^{\mu}}{\partial \tau^{2}}-\frac{\partial^{2} X^{\mu}}{\partial \sigma^{2}}=0 \\
X^{\mu}\left(\tau+\tau_{0}, 2 \pi\right)=X^{\mu}(\tau, 0)+\sum_{k} N_{k} \ell_{k} \delta_{k}^{\mu}, \\
\ddot{X}^{\mu}(\tau, 0)+Q\left[X^{\prime \mu}\left(\tau+\tau_{0}, 2 \pi\right)-X^{\prime \mu}(\tau, 0)\right]=0,
\end{gathered}
$$

дополненной условиями ортонормальности (3). Здесь $Q=\gamma C / m$.

Решение системы (9) было найдено методом Фурье в виде линейной комбинации функций $X^{\mu}(\tau, \sigma)=\alpha^{\mu} T(\tau) u(\sigma)$. Из системы (9) вытекают краевые задачи для функций $T(\tau)$ и $u(\sigma)$ :

$$
\begin{gathered}
T^{\prime \prime}(\tau)+\lambda T=0, \quad T\left(\tau+\tau_{0}\right)=\kappa T(\tau) \\
u^{\prime \prime}(\sigma)+\lambda u=0, \quad u(2 \pi)=\kappa^{-1} u(0), \quad \lambda u(0)=Q\left[\kappa u^{\prime}(2 \pi)-u^{\prime}(0)\right] .
\end{gathered}
$$

Здесь использовано условие замыкания (6) при $N_{k}=0$.

Непосредственная проверка показывает, что решения этих задач сушествуют, если собственные значения $\lambda$ неотрицательны. 
При $\lambda=0$ решение системы (9) есть сумма слагаемых вида $e_{k}^{\mu}\left(a_{k} \tau+b_{k} \sigma\right)$, в которых условие (6) приводит к равенствам

$$
2 \pi b_{k}= \begin{cases}-a_{k} \tau_{0}, & k \leqslant 3, \\ -a_{k} \tau_{0}+\ell_{k} N_{k}, & k>3 .\end{cases}
$$

Здесь единичные векторы $e_{0}, e_{1}, e_{2}, \ldots, e_{D-1}$ образуют ортонормированный базис в $\mathcal{M}$, причем $e_{0}, e_{1}, e_{2}, e_{3}-$ базис в $R^{1,3}$.

В случае $\lambda \equiv \omega^{2}>0$ в задачах (10), (11) параметр $\kappa=e^{ \pm i \omega \tau_{0}}$, а краевая задача (11) (обобщение задачи Штурма-Лиувилля) имеет нетривиальные решения лишь при выполнении условия

$$
\cos 2 \pi \omega-\cos \omega \tau_{0}=\frac{\omega}{2 Q} \sin 2 \pi \omega
$$

Эти решения имеют вид $u_{n}(\sigma)=\sin \omega_{n}(2 \pi-\sigma)+e^{\mp i \omega_{n} \tau_{0}} \sin \omega_{n} \sigma$ и являются собственными функциями задачи (11), отвечающими корням $\omega=\omega_{n}$ уравнения (13).

Итак, найденные собственные функции задач (10) и (11) позволяют представить решение задачи (9) в виде ряда Фурье

$$
\begin{aligned}
X^{\mu}(\tau, \sigma)= & e_{0}^{\mu} a_{0}\left(\tau-\frac{\tau_{0}}{2 \pi} \sigma\right)+\sum_{k \geqslant 3} e_{k}^{\mu}\left(a_{k} \tau+b_{k} \sigma\right)+ \\
& +\sum_{n=-\infty}^{\infty} \alpha_{n}^{\mu}\left[\sin \omega_{n}(2 \pi-\sigma)+e^{i \omega_{n} \tau_{0}} \sin \omega_{n} \sigma\right] e^{-i \omega_{n} \tau} .
\end{aligned}
$$

Здесь предполагается, что $\omega_{-n}=-\omega_{n}, \alpha_{n}^{* \mu}=-\alpha_{-n}^{\mu}$.

Осталось потребовать выполнения условий ортонормальности (3) и равенства

$$
\dot{X}^{2}(\tau, 0)=C^{2},
$$

вытекаюшего из (8), для ряда (14). Это приводит к следуюшей системе уравнений для коэффициентов этого ряда:

$$
\begin{gathered}
a_{0}^{2}\left(1+\frac{\tau_{0}^{2}}{4 \pi^{2}}\right)=\sum_{k \geqslant 3}\left(a_{k}^{2}+b_{k}^{2}\right)-4 \sum_{n>0} \omega_{n}^{2}\left(\alpha_{n}, \alpha_{n}^{*}\right)\left(1-\cos \omega_{n} \tau_{0} \cdot \cos 2 \pi \omega_{n}\right), \\
\frac{\tau_{0}}{2 \pi} a_{0}^{2}+\sum_{k \geqslant 3} a_{k} b_{k}+2 \sum_{n>0} \omega_{n}^{2}\left(\alpha_{n}, \alpha_{n}^{*}\right) \sin \omega_{n} \tau_{0} \cdot \sin 2 \pi \omega_{n}=0 \\
a_{0}^{2}-\sum_{k \geqslant 3} a_{k}^{2}+2 \sum_{n>0} \omega_{n}^{2}\left(\alpha_{n}, \alpha_{n}^{*}\right) \sin ^{2} 2 \pi \omega_{n}=C^{2} \\
N_{k}\left(e_{k}, \alpha_{n}\right)=\left(e_{0}, \alpha_{n}\right)=0, \quad\left(\alpha_{m}, \alpha_{n}\right)=0, \quad m \neq-n .
\end{gathered}
$$

Уравнения (13) и (15) образуют систему, определяюшую значения параметров, (например, $\tau_{0}$ и $\left.\omega_{n}\right)$ для ряда (14). Решения (14), содержашие единственную ненулевую частоту, будем называть одночастотными.

В рассматриваемом многообразии $\mathcal{M}$ даже в простейшем случае одночастотных решений при $\tau_{0}=0$ сушествуют топологически нетривиальные (отсутствуюшие в $R^{1,3}$ ) 
решения, описывающие движение замкнутой струны, не стягиваемой в точку. Здесь под струной подразумевается сечение $t=$ const мировой поверхности (14). Безразмерные частоты $\omega=\omega_{n}$ этих состояний при $\tau_{0}=0$ являются корнями уравнения

$$
\sin \pi \omega \cdot\left(\sin \pi \omega+\frac{\omega}{2 Q} \cos \pi \omega\right)=0
$$

Корням уравнения (16) $\omega_{n}=n$ отвечают нетривиальные решения

$$
X^{\mu}=e_{0}^{\mu} a_{0} \tau+\sum_{k>3} e_{k}^{\mu} b_{k} \sigma+A_{n} \sin n \sigma \cdot e^{\mu}(n \tau),
$$

где

$$
e^{\mu}(n \tau)=e_{1}^{\mu} \cos n \tau+e_{2}^{\mu} \sin n \tau, \quad a_{0}=\sqrt{n^{2} A_{n}^{2}+\sum_{k} b_{k}^{2}}, \quad b_{k}=\frac{\ell_{k} N_{k}}{2 \pi} .
$$

Здесь мы положили $a_{k}=0$ для $k \geqslant 3$ без потери обшности, так как слагаемые, содержащие $a_{k}, k \geqslant 1$, в выражении (14) описывают прямолинейное равномерное движение системы с постоянной скоростью. Эти слагаемые можно обратить в нуль с помощью преобразования Лоренца.

Решения (17) описьвают равномерное врашение в плоскости $e_{1}, e_{2}$ струны, имеющей форму синусоиды, длина которой конечна ввиду цикличности координаты с ортом $e_{k}$. Массивная точка покоится (при $\sigma=0$ ) или отсутствует.

Корням $\omega_{n}=-2 Q \operatorname{tg} \omega_{n} \neq n$, обращающим в нуль второй сомножитель уравнения (16), соответствуют ротационные движения

$$
X^{\mu}=e_{0}^{\mu} a_{0} \tau+\sum_{k>3} e_{k}^{\mu} b_{k} \sigma+A_{n} \cos \left[\omega_{n}(\sigma-\pi)\right] \cdot e^{\mu}\left(\omega_{n} \tau\right) .
$$

Они подобны движениям (17), но масса $m$ (в точке $\sigma=0$ ) движется по окружности вместе с врашаюшейся струной. Два отрезка струны, удерживающие эту массу, присоединены к ней под углом $\beta<\pi$.

В случае $b_{k}=0$ уравнения $(17),(18)$ описывают в пространстве Минковского $R^{1,3}$ равномерное врашение сложенной вдвое (и после этого - $(n-1)$ раз) струны. Такие состояния были классифицированы в работе [8]. Они характеризуются наличием точек возврата струны, движушихся со скоростью света (с особенностями метрики $\dot{X}^{2}=$ $\left.X^{\prime 2}=0\right)$. В то же время решения (17) и (18) при $b_{k} \neq 0$, описываюшие врашение не стягиваемой в точку струны в $\mathcal{M}$, не имеют таких особенностей.

Одночастотные решения (14) в случае $\tau_{0} \neq 0$ представимы в виде

$$
\begin{aligned}
X^{\mu}= & e_{0}^{\mu} a_{0}\left(\tau-\frac{\tau_{0}}{2 \pi} \sigma\right)+\sum_{k>3} e_{k}^{\mu} \frac{\ell_{k} N_{k}}{2 \pi} \sigma+ \\
& +A_{n}\left\{f_{n}(\sigma) \cdot e^{\mu}\left(\omega_{n} \tau\right)-\sin \omega_{n} \tau_{0} \cdot \sin \omega_{n} \sigma \cdot e^{\mu}\left(\omega_{n} \tau\right)\right\},
\end{aligned}
$$

где

$$
\dot{e}^{\mu}\left(\omega_{n} \tau\right)=-e_{1}^{\mu} \sin \omega_{n} \tau+e_{2}^{\mu} \cos \omega_{n} \tau, \quad f_{n}(\sigma)=\sin \omega_{n}(2 \pi-\sigma)+\cos \omega_{n} \tau_{0} \cdot \sin \omega_{n} \sigma .
$$


Значения $\tau_{0}, \omega_{n}, a_{0}, A_{n}=\sqrt{-2\left(\alpha_{n}, \alpha_{n}^{*}\right)}$ определяются из системы уравнений (13), (15); $\alpha_{n}^{\mu}=A_{n}\left(e_{1}^{\mu}+i e_{2}^{\mu}\right) / 2 ;$ коэффициенты $b_{k}$ заданы выражением (12).

Из системы (15) следует уравнение

$$
1+\theta^{2}-2 \theta \frac{1-\cos \omega_{n} \tau_{0} \cdot \cos 2 \pi \omega_{n}}{\sin \omega_{n} \tau_{0} \cdot \sin 2 \pi \omega_{n}}=\left(1-\theta \frac{\sin 2 \pi \omega_{n}}{\sin \omega_{n} \tau_{0}}\right) \frac{\gamma^{2}}{m^{2} Q^{2}} \sum_{k>3} b_{k}^{2} .
$$

Здесь и ниже $\theta=\tau_{0} /(2 \pi)$. Система уравнений $(13),(20)$ определяет спектр значений $\tau_{0}$ и $\omega_{n}$ независимо от амплитудных множителей $a_{0}$ и $A_{n}$.

При $b_{k}=N_{k}=0$ для любого $k \geqslant 3$ уравнение (20) имеет вид

$$
\frac{\tau_{0}}{2 \pi}+\frac{2 \pi}{\tau_{0}}=2 \frac{1-\cos \omega_{n} \tau_{0} \cdot \cos 2 \pi \omega_{n}}{\sin \omega_{n} \tau_{0} \cdot \sin 2 \pi \omega_{n}} .
$$

В случае $b_{k}=0$ решения (19) описывают равномерное врашение замкнутой струны, имеющей форму гипоциклоиды, соединенной под ненулевым углом в массивной точке. Эта форма является сечением $t=t_{0}=$ const мировой поверхности (19):

$$
\begin{aligned}
\left.X^{\mu}\right|_{t=t_{0}}= & A_{n}\left\{\left(f_{n} \cos \omega_{n} \theta \sigma+\sin \omega_{n} \tau_{0} \cdot \sin \omega_{n} \sigma \cdot \sin \omega_{n} \theta \sigma\right) e^{\mu}\left(\frac{\omega_{n} t_{0}}{a_{0}}\right)+\right. \\
& \left.+\left(f_{n} \sin \omega_{n} \theta \sigma-\sin \omega_{n} \tau_{0} \cdot \sin \omega_{n} \sigma \cdot \cos \omega_{n} \theta \sigma\right) \hat{e}^{\mu}\left(\frac{\omega_{n} t_{0}}{a_{0}}\right)\right\}+e_{0}^{\mu} t_{0} .
\end{aligned}
$$

Гипоциклоида есть траектория точки окружности, катящейся внутри неподвижной окружности большего радиуса. Обозначив радиусы этих окружностей соответственно $r$ и $R$, представим уравнение гипоциклоиды в виде

$$
\begin{aligned}
& x(t)=(R-r) \cos \frac{r}{R} t+r \cos \frac{R-r}{R} t, \\
& y(t)=(R-r) \sin \frac{r}{R} t-r \sin \frac{R-r}{R} t .
\end{aligned}
$$

Преобразуем правую часть (22) к виду

$$
\begin{aligned}
& A_{n}\left\{\left(\sin \pi \omega_{n}(1+\theta) \cdot \cos \omega_{n}(1-\theta) \sigma+\sin \pi \omega_{n}(1-\theta) \cdot \cos \omega_{n}(1+\theta) \sigma\right) e^{\mu}+\right. \\
& \left.\quad+\left(\sin \pi \omega_{n}(1+\theta) \cdot \sin \omega_{n}(1-\theta) \sigma-\sin \pi \omega_{n}(1-\theta) \cdot \sin \omega_{n}(1+\theta) \sigma\right) e^{\mu}\right\}+e_{0}^{\mu} t_{0} .
\end{aligned}
$$

Сравнивая это выражение с уравнениями (23), находим, что линия (22) является гипоциклоидой, если имеет место равенство

$$
\frac{\sin \pi \omega_{n}(1-\theta)}{\sin \pi \omega_{n}(1+\theta)}=\frac{1-\theta}{1+\theta} .
$$

Оно равносильно условию (21), которое, напомним, ограничивает возможные значения параметров $\omega_{n}$ и $\theta$ в случае $a_{k}=b_{k}=0$ при $k \geqslant 3$. Следовательно, при выполнении условия (21) линия (22) является гипоциклоидой с отношением радиусов окружностей

$$
\frac{r}{R}=\frac{1-|\theta|}{2}
$$


При этом $|\theta|<1$, что следует также из принципа причинности.

Данная гипоциклоида вращается в плоскости $e_{1}, e_{2}$ с угловой скоростью $\Omega=\omega_{n} / a_{0}$, где $a_{0}$ определяется уравнениями (15):

$$
\begin{gathered}
a_{0}^{2}=\frac{m^{2} Q^{2}}{\gamma^{2}\left(1-v^{2}\right)} \\
v^{2}=\left(\frac{A_{n} \omega_{n} \sin 2 \pi \omega_{n}}{a_{0}}\right)^{2}=\theta \frac{\sin 2 \pi \omega_{n}}{\sin \omega_{n} \tau_{0}} .
\end{gathered}
$$

Массивная точка движется со скоростью $v$ по окружности радиуса $v / \Omega$. Врашающаяся гипоциклоида (22) имеет точки возврата, которые движутся со скоростью света. Подобные решения для струнной модели “треугольник” были впервые получены в работе [4].

Одночастотные решения (19), включаюшие слагаемые с $b_{k} \neq 0$ (для циклических координат в $\mathcal{M}$ ), описывают вращающиеся пространственные кривые, не имеющие точек возврата. Последнее означает, что на мировых поверхностях (19) при $b_{k} \neq 0$ отсутствуют особенности $\dot{X}^{2}=X^{\prime 2}=0$.

Существуют различные топологические типы решений (19), различающиеся как гомотопическим классом замкнутой струны (набором чисел $N_{k}, k>3$ ), так и видом проекции струны на плоскость $e_{1}, e_{2}$. В частности, при $b_{k}=0$ данная проекция (22) (гипоциклоида) может иметь различное число точек возврата.

В основу классификации решений (19) положим подход, использованный ранее для описания различных топологических состояний струнной модели бариона “треугольник" [9]. А именно, при фиксированных значениях чисел $N_{k}, \ell_{k}$ и параметров $\gamma, a_{0}$ для заданного топологического типа решения (19) рассмотрим предельный переход $m \rightarrow 0$.

Анализ уравнений (13), (15), (19), (24), (25) показывает, что при $m \rightarrow 0$ величина $Q$ стремится к бесконечности, а значения параметров $2 \omega$ и $\tau_{0} \omega / \pi$ стремятся к целым числам, для которых мы введем следуюшие обозначения:

$$
n_{1}=\left|\lim _{m \rightarrow 0} 2 \omega\right|, \quad n_{2}=\lim _{m \rightarrow 0} \frac{\tau_{0} \omega}{\pi}=\lim _{m \rightarrow 0} 2 \theta \omega .
$$

В силу неравенства $|\theta|<1$ и условия $(13)$, приводящего к равенству $(-1)^{n_{1}}=(-1)^{n_{2}}$ ( $n_{1}$ и $n_{2}$ имеют одинаковую четность), допустимы лишь следуюшие значения параметров (26):

$$
n_{1} \geqslant 2, \quad n_{2}=n_{1}-2, n_{1}-4, \ldots,-\left(n_{1}-2\right) .
$$

Отметим, что среди решений с $n_{2}=0$, которым соответствует $\tau_{0}=0$, в случае $m \neq 0$ следует различать два класса - состояния (17) и (18) с покоящейся и движущейся массивной точкой, соответственно. В пределе $m \rightarrow 0$ решения обоих классов стремятся к одинаковому предельному решению, описывающему вращение сложенной замкнутой струны без масс.

Если учесть последнее обстоятельство, то можно сделать вывод, что набор целых чисел $N_{k}, n_{1}, n_{2}$, ограниченных условиями $(27)$, однозначно определяет топологический тип одночастотных состояний $(17),(18)$ или (19) замкнутой струны с массивной точкой 
в пространстве $\mathcal{M}$. Фигурировавший в выражениях (17)-(19) индекс $n$ можно воспринимать как мультииндекс: $n=\left(n_{1}, n_{2}\right)$. Он относится не только к $\omega_{n}, A_{n}$, но и к параметрам $\theta, a_{0}$, которые также связаны с топологическим типом состояния.

По своему геометрическому смыслу параметр $n_{1}$ равен числу точек возврата гипоциклоиды, описываемой выражением $(22)$ в пределе $m \rightarrow 0, \ell_{k} \rightarrow 0$, с учетом их кратности при изменении $\sigma$ от 0 до $2 \pi$. Параметр $n_{2}$ характеризует форму данной гипоциклоиды, например для $n_{1}=5$ при $n_{2}=3$ получается криволинейный пятиугольник, а значению $n_{2}=1$ соответствует звезда.

Возможные приложения найденных решений для рассматриваемой модели связаны с описанием внутренних степеней свободы элементарных частиц с заданным набором квантовых чисел и физических характеристик. Вычислим важнейшие из них - энергию $E$ и угловой момент $J$ одночастотных состояний (14).

Для произвольного состояния замкнутой релятивистской струны, нагруженной точечной массой, энергия и угловой момент определяются с помошью следуюших интегралов (токов Нётер) [2], [10]:

$$
\begin{aligned}
P^{\mu} & =\int_{\mathcal{C}} p^{\mu}(\tau, \sigma) d \sigma+p_{1}^{\mu}(\tau), \quad p^{\mu}(\tau, \sigma)=\gamma \frac{\left(\dot{X}, X^{\prime}\right) X^{\prime \mu}-X^{\prime 2} \dot{X}^{\mu}}{\left[\left(\dot{X}, X^{\prime}\right)^{2}-\dot{X}^{2} X^{\prime 2}\right]^{1 / 2}}, \\
\mathcal{J}^{\mu \nu} & =\int_{\mathcal{C}}\left[X^{\mu} p^{\nu}-X^{\nu} p^{\mu}\right] d \sigma+\left[x_{1}^{\mu}(\tau) p_{1}^{\nu}(\tau)-x_{1}^{\nu}(\tau) p_{1}^{\mu}(\tau)\right],
\end{aligned}
$$

где $x_{1}^{\mu}(\tau)=X^{\mu}\left(\tau, \sigma_{1}(\tau)\right)=X^{\mu}(\tau, 0)$ и $p_{1}^{\mu}(\tau)=m \dot{x}_{1}^{\mu}(\tau) / \sqrt{\dot{x}_{1}^{2}(\tau)}$ - координаты и импульс массивной точки, $\mathcal{C}$ - любой замкнутый контур, охватываюший трубкообразную мировую поверхность струны.

Линии $\tau=$ const на мировой поверхности (19) при $\tau_{0} \neq 0$ незамкнуты, поэтому они не могут служить контуром интегрирования. В качестве контура $\mathcal{C}$ в интегралах $(28),(29)$ нужно использовать другие линии, например $\tau-\theta \sigma=$ const (т.e. $t=$ const).

Вычисление интегралов (28), (29) удобно проводить, сделав замену координат $\tilde{\tau}=$ $\tau-\theta \sigma, \tilde{\sigma}=\sigma-\theta \tau$, при которой сохраняются условия ортонормальности (3). В ортонормальной калибровке $(3) p^{\mu}(\tau, \sigma)=\gamma \dot{X}^{\mu}(\tau, \sigma)$.

Если мы подставим выражения (19) (включая частные случаи $(17),(18)$ ) в уравнения (28), то получим следуюшее выражение для импульса:

$$
P^{\mu}=\gamma\left\{a_{0}\left[2 \pi\left(1-\theta^{2}\right)+Q^{-1}\right] e_{0}^{\mu}+\theta \sum_{k>3} \ell_{k} N_{k} e_{k}^{\mu}\right\} .
$$

Квадрат энергии равен скалярному квадрату сохраняюшегося вектора импульса (28) или (30): $E^{2}=P^{2}=P_{\mu} P^{\mu}$.

Угловой момент (29) не является постоянным для заданной системы из-за анизотропии пространства $\mathcal{M}$. Лишь компоненты $\mathcal{J}^{\mu \nu}$ с $\mu, \nu=0,1,2,3$, относяшиеся к пространству $R^{1,3}$, постоянны. Из них только $z$-компонента углового момента отлична от нуля:

$$
\begin{gathered}
\mathcal{J}^{\mu \nu}=j_{3}^{\mu \nu} J, \\
J=\frac{\gamma}{2 \omega_{n}}\left\{2 \pi\left[a_{0}^{2}\left(1-\theta^{2}\right)-\sum_{k>3} b_{k}^{2}\right]+\frac{a_{0}^{2}}{Q}-\frac{m^{2} Q}{\gamma^{2}}\right\},
\end{gathered}
$$


где $j_{3}^{\mu \nu}=e_{1}^{\mu} e_{2}^{\nu}-e_{1}^{\nu} e_{2}^{\mu}=e^{\mu} e^{\nu}-e^{\nu} e^{\mu}$.

Выражения (30) и (31) для импульса и углового момента одночастотных состояний (14) можно обобщить на случай $a_{k} \neq 0$. При этом импульс $P^{\mu}$ и момент $J$ следует дополнить слагаемыми соответственно

$$
\Delta P^{\mu}=\gamma\left[2 \pi\left(1-\theta^{2}\right)+Q^{-1}\right] \sum_{k>3} a_{k} e_{k}^{\mu}, \quad \Delta J=-\frac{\gamma}{2 \omega_{n}}\left[2 \pi\left(1-\theta^{2}\right)+Q^{-1}\right] \sum_{k>3} a_{k}^{2} .
$$

Тем не менее ниже мы будем полагать $a_{k}=0$, что, как упоминалось ранее, не ведет к потере общности.

В частном случае $N_{k}=0$ значения $E$ и $J$ связаны условием

$$
J=\frac{1}{2 \Omega}\left(E-m \sqrt{1-v^{2}}\right)=\frac{a_{0}}{2 \omega_{n}}\left(E-m \sqrt{1-v^{2}}\right) .
$$

Здесь $v$-постоянная скорость массивной точки.

Соотношения $(30),(31)$ задают неявную зависимость $J=J\left(E^{2}\right)$ классического углового момента от квадрата энергии для одночастотного состояния определенного топологического типа. Если фиксирован топологический тип, определяемый числами $n_{1}$, $n_{2}, N_{k}$, а также значения $m$ и $\gamma$, то состояния (17)-(19) образуют однопараметрическое множество. В качестве параметра данного множества можно использовать любую переменную из следуюшего набора: $\omega_{n}, \tau_{0}$ (или $\left.\theta=\tau_{0} /(2 \pi)\right), Q, v, a_{0}, A_{n}, E, J$ и др. Остальные параметры выражаются через один заданный с помощью формул (13), (20), (24), (25), (30), (31).

График получаюшейся при этом зависимости $J=J\left(E^{2}\right)$ (траектория Редже) зависит от выбранного топологического типа одночастотного состояния. В качестве примера на рисунке показаны траектории Редже для состояний (17)-(19), полученные с помощью численных расчетов. Здесь $\gamma=1 /(2 \pi), m=1 / 2$ для линий $1-4$ и $m=3$ для линии 5.

Топологический тип для каждой из траекторий Редже на рисунке иллюстрируется формой струны (22) - сечения $t=$ const мировой поверхности. В частности, для кривых 1 и 5 (различающихся лишш значением массы $m$ ) проекция этого сечения на плоскость $e_{1}, e_{2}$ есть прямолинейньй отрезок с массой $m$ на конце (что отвечает $n_{1}=2$, $\left.n_{2}=0\right)$; для траектории 2 - криволинейный треугольник с $n_{1}=3, n_{2}=1$; для траектории 3 - кривая, близкая к астроиде с $n_{1}=4, n_{2}=1$; для траектории 4 - "звезда" с $n_{1}=5, n_{2}=1$. Сплошные линии отвечают значению $\sum_{k} b_{k}^{2}=0$, тонкие пунктирные значению $\sum_{k} b_{k}^{2}=1$. Отметим, что параметр $\sum_{k} b_{k}^{2}=(2 \pi)^{-2} \sum_{k} \ell_{k}^{2} N_{k}^{2}$ по своему физическому смыслу должен быть мал по сравнению с квадратом размера струны $A_{n}^{2}$.

Для каждой из траекторий значения параметра $\omega_{n}$, используемого для параметризации кривой, пробегают интервал, определяемый неравенством $n_{1}-1<2 \omega_{n}<n_{1}$. Для каждого значения $\omega_{n}$ из данного интервала с помощью численного решения уравнений (13), (20) найдены величины $\theta$ и $Q$, а далее - значения $v, a_{0}, E, J$ с помошью формул (25), (24), (30), (31).

Предел $\omega_{n} \rightarrow n_{1} / 2-0$ для всех траекторий Редже соответствует $E \rightarrow \infty$, в то время как в противоположном пределе $\omega_{n} \rightarrow\left(n_{1}-1\right) / 2+0$ для траекторий $1,2,4,5$ на рисунке массивная точка стремится к центру вращения, а величины $E$ и $J$ - к минимальным значениям для данной траектории. Однако на V-образной траектории 3 пределу 


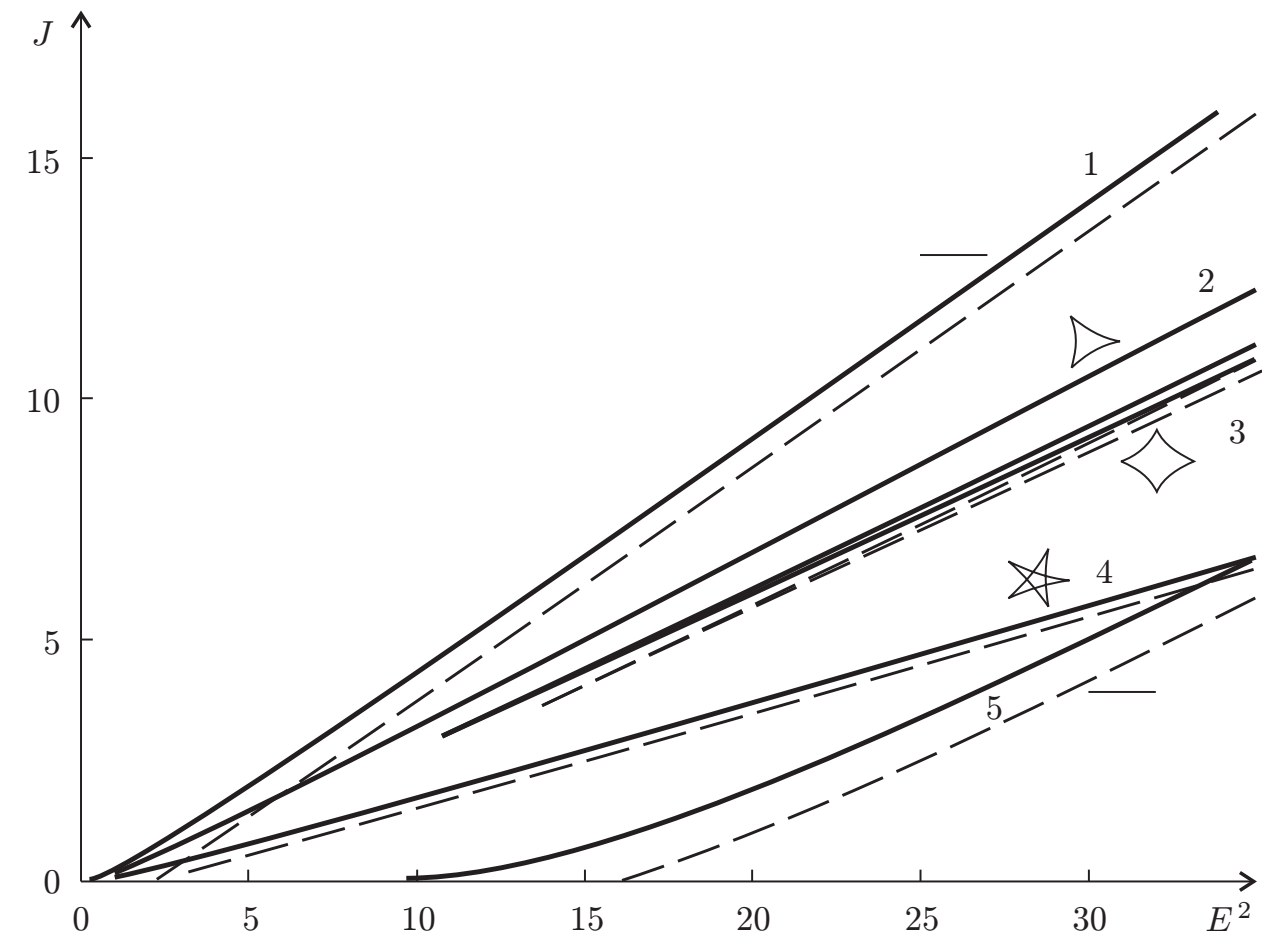

Траектории Редже для одночастотных состояний

$\omega_{n} \rightarrow\left(n_{1}-1\right) / 2+0$ отвечают верхняя ветвь этой кривой и неограниченное возрастание $E$ и $J$ (массивная точка при этом приближается к середине звена кривой, стремяшейся к трехзвенной гипоциклоиде).

Траектории Редже для состояний (17)-(19) рассматриваемой модели близки к прямолинейным (переходят в прямолинейные в случае $m=0$ ). В наибольшей степени это относится к области энергий $E \gg m$.

Рассмотрим предел $E \rightarrow \infty$, соответствующий пределу $\omega_{n} \rightarrow n_{1} / 2-0$ и реализуюшийся для всех топологических типов одночастотных решений за исключением решений (17). В данном ультрарелятивистском пределе скорость массивной точки стремится к скорости света, $v \rightarrow 1$, и имеют место соотношения (26), что позволяет исследовать асимптотическое поведение состояний (17)-(19), введя малые параметры $\varepsilon, \varepsilon_{i}$ такие, что

$$
\varepsilon=\sqrt{1-v^{2}}, \quad 2 \omega=n_{1}-\varepsilon_{1}, \quad n_{1} \theta=n_{2}-\varepsilon_{2} .
$$

Подставив эти соотношения в уравнения $(13),(20),(25)$, выразим через один из малых параметров остальные:

$$
\varepsilon_{1} \simeq \frac{2 n_{1}}{\pi \nu} \varepsilon+\frac{n_{1}^{3}}{3 \pi \nu^{3}} \varepsilon^{3}, \quad \varepsilon_{2} \simeq \frac{2 n_{2}}{3 \pi \nu} \varepsilon^{3}+\frac{n_{2}\left(n_{1}^{2}-6 n_{2}^{2}\right)}{3 \pi \nu^{3}} \varepsilon^{5},
$$

где $\nu=\sqrt{n_{1}^{2}-n_{2}^{2}}$. Эти разложения после подстановки в формулы $(24),(30),(31)$ приводят к следуюшим асимптотическим выражениям для энергии и углового момента в 
случае $a_{k}=0$ :

$$
\begin{aligned}
E^{2} & \simeq \frac{\pi^{2} \nu^{2} m^{2}}{4}\left(\frac{1}{\varepsilon^{2}}-\frac{n_{1}^{2}-2 n_{2}^{2}}{2 \nu^{2}}\right)^{2}+\frac{\pi m^{2}\left(n_{1}^{2}-2 n_{2}^{2}\right)}{3 \nu} \cdot \frac{1}{\varepsilon}-\gamma^{2} \frac{n_{2}^{2}}{n_{1}^{2}} \sum_{k>3} b_{k}^{2}, \\
J & \simeq \frac{\pi n_{1} m^{2}}{8 \gamma}\left(\frac{1}{\varepsilon^{4}}-\frac{n_{1}^{2}-2 n_{2}^{2}}{\nu^{2}} \cdot \frac{1}{\varepsilon^{2}}+\frac{4 n_{1}^{2}}{3 \nu^{3}} \cdot \frac{1}{\varepsilon}\right)-\frac{2 \pi \gamma}{n_{1}} \sum_{k>3} b_{k}^{2} .
\end{aligned}
$$

Используя данные разложения, находим в пределе $v \rightarrow 1, E \rightarrow \infty, J \rightarrow \infty$ асимптотическое выражение для зависимости $J$ от $E^{2}$ в случае состояния произвольного типа с заданными значениями параметров $n_{1}, n_{2}, b_{k}$ :

$$
J \simeq \alpha^{\prime} E^{2}+\alpha_{1} E^{1 / 2}+\alpha_{0}, \quad E \rightarrow \infty,
$$

где

$$
\alpha^{\prime}=\frac{1}{2 \pi \gamma} \frac{n_{1}}{n_{1}^{2}-n_{2}^{2}}, \quad \alpha_{1}=-\frac{\sqrt{2} n_{1} m^{3 / 2}}{3 \sqrt{\pi} \gamma \nu^{3 / 2}}, \quad \alpha_{0}=-\frac{2 \pi \gamma\left(n_{1}^{2}-2 n_{2}^{2}\right)}{n_{1} \nu^{2}} \sum_{k>3} b_{k}^{2} .
$$

Эта зависимость близка к линейному виду [2], [5] $J=\alpha^{\prime} E^{2}+\alpha_{0}$, однако коэффициент наклона $\alpha^{\prime}$ для данной модели отличается от значения Намбу $\alpha^{\prime}=1 /(2 \pi \gamma)$ множителем $n_{1} / \nu^{2}=n_{1} /\left(n_{1}^{2}-n_{2}^{2}\right)$, зависяшим от топологического типа (см. рисунок). Для ротационных движений (17), (18) топологического типа $n_{1}=2, n_{2}=0$ (траектории 1 на рисунке) этот множитель достигает максимального возможного значения $1 / 2$.

Для физических приложений найденных состояний (14)-(19) замкнутой струны с точечной массой необходимо их квантовое описание. Эта процедура, сушественно осложненная нелинейностью уравнения ( 7$)$ при $m \neq 0$, выходит за рамки данной статьи.

\section{Список литературы}

[1] М. Грин, Дж. Швари, Э. Виттен. Теория суперструн. М.: Мир, 1990.

[2] Б. М. Барбашов, В. В. Нестеренко. Модель релятивистской струны в физике адронов. М.: Энергоатомиздат, 1987.

[3] И. Н. Никитин. ТМФ. 1996. Т. 109. № 2. С. 202; hep-th/0401002.

[4] Г. С. Шаров. ТМФ. 1997. Т. 113. № 1. С. 68.

[5] Г. С. Шаров. ЯФ. 1999. T. 62. № 10. C. 1831; A. Inopin, G. S. Sharov. Phys. Rev. D. 2001. V. 63. № 5. P. 054023.

[6] G. S. Sharov. Phys. Rev. D. 2000. V. 62. № 9. P. 094015; hep-ph/0004003.

[7] С. В. Талалов. ТМФ. 2003. Т. 135. № 2. С. 289.

[8] В. П. Петров, Г. С. Шаров. ТМФ. 1996. Т. 109. № 2. С. 187.

[9] Г. С. Шаров. ТМФ. 1998. Т. 114. № 2. С. 277.

[10] G. S. Sharov. Exited states of rotating relativistic string with massive ends. hep-ph/0311060.

Поступила в редакцию 22.III.2004 г. 